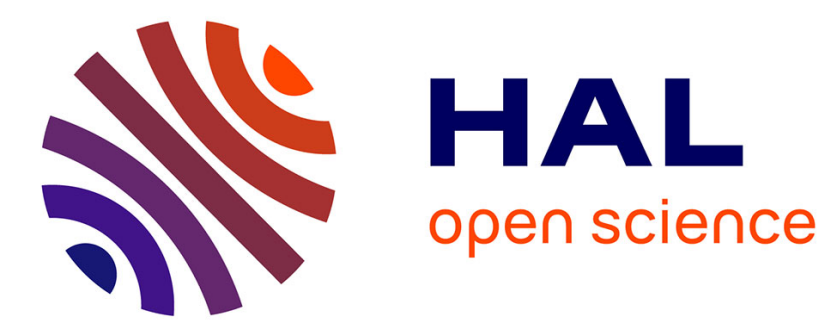

\title{
Spatio-temporal segmentation and estimation of ocean surface currents from satellite sea surface temperature fields
}

\author{
Pierre Tandeo, Sileye Ba, Ronan Fablet, Bertrand Chapron, Emmanuelle \\ Autret
}

\section{To cite this version:}

Pierre Tandeo, Sileye Ba, Ronan Fablet, Bertrand Chapron, Emmanuelle Autret. Spatio-temporal segmentation and estimation of ocean surface currents from satellite sea surface temperature fields. ICIP 2013 : 20 th IEEE International Conference on Image Processing, Sep 2013, Melbourne, Australia. pp.2344 - 2348, 10.1109/CIP.2013.6738483 . hal-00946865

\section{HAL Id: hal-00946865 https://hal.science/hal-00946865}

Submitted on 14 Feb 2014

HAL is a multi-disciplinary open access archive for the deposit and dissemination of scientific research documents, whether they are published or not. The documents may come from teaching and research institutions in France or abroad, or from public or private research centers.
L'archive ouverte pluridisciplinaire HAL, est destinée au dépôt et à la diffusion de documents scientifiques de niveau recherche, publiés ou non, émanant des établissements d'enseignement et de recherche français ou étrangers, des laboratoires publics ou privés. 


\section{SPATIO-TEMPORAL SEGMENTATION AND ESTIMATION OF OCEAN SURFACE CURRENTS FROM SATELLITE SEA SURFACE TEMPERATURE FIELDS}

\author{
Pierre Tandeo, Sileye Ba, Ronan Fablet*
}

\author{
Telecom Bretagne \\ Department Signal \& Communications \\ Technopôle Brest-Iroise, France
}

Bertrand Chapron, Emmanuelle Autret

\author{
IFREMER \\ Spatial Oceanography Laboratory \\ Technopôle Brest-Iroise, France
}

\begin{abstract}
The use of satellite Sea Surface Temperature (SST) fields to retrieve zonal and meridional surface currents $(\mathrm{U}, \mathrm{V})$ is now a widespread idea. Since the classical approach involves temporal differencing of SST fields, we investigate in this paper the extent to which mesoscale ocean dynamics may be decomposed into a superposition of dynamical modes, characterized by different linear relationships between surface currents and temperature fields. Based on a completely observation-driven approach, we propose a latent class regression model from local satellite surface currents and patches of SST measurements. Applied to the highly dynamical Agulhas region, we demonstrate and discuss the geophysical relevance of the proposed mixture model to achieve a spatio-temporal segmentation and tracking of the ocean surface dynamical modes. Moreover, we show the accuracy of the proposed model to predict mesoscale surface currents from SST single maps.
\end{abstract}

Index Terms - Observation-driven model, Patch-based model, Latent class regression, Surface dynamical mode segmentation, Sea surface current

\section{INTRODUCTION}

Since the last two decades, satellite microwave sensors have been providing an important source of spatial and temporal information of Sea Surface Temperature (SST). By contrast, satellite altimeters provide very sparse measurements of zonal and meridional surface currents $(\mathrm{U}, \mathrm{V})$ and therefore mesoscale structures with horizontal scales of $50 \mathrm{~km}$ to few hundred kilometers. The classical approach to fill in missing data in the altimetrer data relies on a temporal differencing of SST fields as in the maximum cross-correlation technique or alternate strategies (see e.g. [1], [2], [3]). Alternatively, recent studies (cf. [4], [5], [6], [7], [8]) rationalize and demonstrate that fields of SST can become, to some assumptions, an active tracer coupled to the dynamics leading to strong correlations with surface current fields. This is known as the Surface

\footnotetext{
*Thanks to AVISO and RSS projects for providing data.
}

Quasi-Geostrophic (SQG) theory given in Eq. (1). Within this theoretical framework, a linear transfer function shall be identified between $(\mathrm{U}, \mathrm{V})$ and SST gradient fields. This strongly advocates for observation-driven studies to explore and characterize the local relationships between SST and surface currents from satellite-based routine observations. Yet, as illustrated in Fig. 1, a single linear transfer function cannot be expected to solely govern the whole mesoscale dynamics in a particular ocean region. As revealed, an overall spatial correlation exists, but this relationship clearly varies spatially and temporally. For instance, in the warmer SST frontal zone (top of the image), SST gradients correspond to large surface currents. In the colder frontal area (bottom of the image), large SST gradients do not reflect in large surface currents. We may also point out that within the clearly detected eddy (top-left of the image), significant velocities are associated with weak SST gradients.

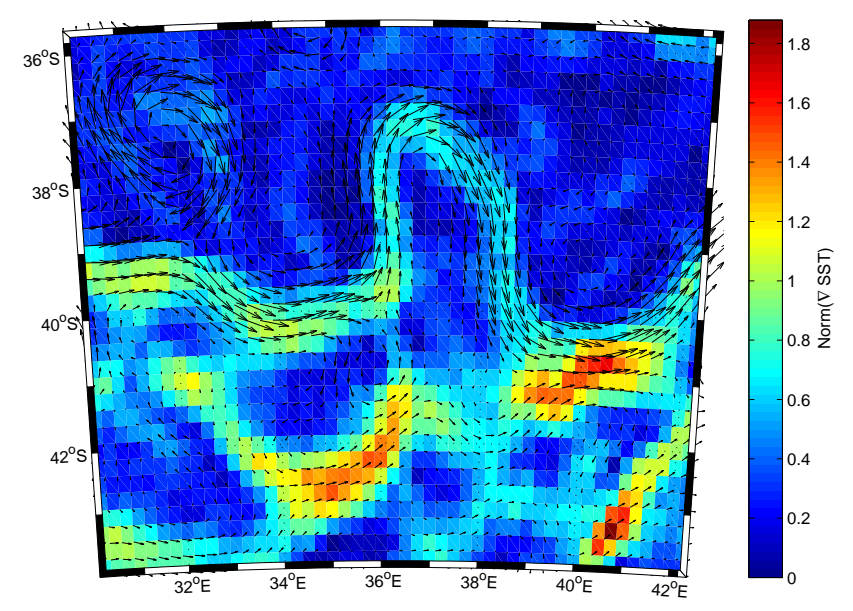

Fig. 1. Surface currents and associated temperature gradient norms derived from satellite measurements within the Agulhas current the $1^{\text {st }}$ of January, 2004.

Within an observation-driven framework, the key statement of the proposed approach is to regard the local dynamics as a mixture of different dynamical modes, i.e., local dynam- 
ics are stated as the superimposition of a finite set of linear transfer functions. In this paper, we propose to investigate such a model to (i) develop a probabilistic learning-based setting for the inference of such mixture models, (ii) achieve a spatio-temporal segmentation of the identified dynamical modes from satellite SST and surface currents observations, (iii) evaluate the extent to which such mixture models are geophysically relevant to characterize the upper ocean dynamics over active ocean regions, and to (iv) analyze their relevance for the estimations of mesoscale surface currents from SST fields.

Hereafter we consider the Agulhas region as our casestudy region and the paper is organized as follows. Section 2 presents the proposed probabilistic learning-based model. In Section 3, the application to satellite observations is evaluated. We further discuss and summarize the key results of our investigations in Section 4.

\section{METHODS}

\subsection{Patch-based approach}

As mentionned above, the SQG model has been shown in recent studies to match the observed 3D dynamics of the ocean surface layer (cf. [7]). In the SQG model, these dynamics are fully governed by the surface dynamics and the temperature becomes an active tracer which is intrinsically linked to the surface current (cf. [9]) as follows:

$$
(\mathrm{U}, \mathrm{V})=\gamma \nabla^{\perp} \Delta^{-1 / 2} \mathrm{SST}
$$

where $\gamma$ is a positive scalar, $\nabla^{\perp}$ is the orthogonal gradient and $\Delta^{-1 / 2}$ is the fractional Laplacian operator with dimension $-1 / 2$ (cf. [10]). This equation states that the surface current is a linear transform of spatial derivatives of the SST. The parameter $\gamma$ relates to a normalization constraint. Though this parameter is generally assumed to be locally constant, it may not be the case for an entire region as illustrated in Fig. 1. These considerations lead us to hypothesize that surface currents may locally relate to SST derivatives according to a finite set of $K$ transfer functions such as

$$
(\mathrm{U}, \mathrm{V})=\mathcal{F}_{k}(\mathrm{SST})
$$

where $\mathcal{F}_{k}$ characterizes the $k^{t h}$ mode of local interaction between the SST and the surface currents. In the SQG setting expressed in Eq. (1), it may be noticed that, given finitedifference approximations of these derivatives, the transfer function numerically resorts the local convolution of the SST by a linear filter. Therefore we assume that each dynamical mode $\mathcal{F}_{k}$ is parameterized by a linear filter. Using a matricial formulation, Eq. (2) is rewritten as a patch-based linear regression such as

$$
\mathbf{Y}_{i}=\mathbf{H}_{k} \mathbf{X}_{i}
$$

where $\mathbf{Y}_{i}$ encoded as a 2-dimensional vector given by the surface current $(\mathrm{U}, \mathrm{V})$ at spatio-temporal location $i$ and $\mathbf{X}_{i}$ is the vectorized version of the local SST centered in $i$ (cf. Fig. 2). The $p \times 2$ regression coefficient matrix $\mathbf{H}_{k}$ associated with dynamical mode $k$ is corresponding to $\mathcal{F}_{k}$ in Eq. (2). Here, $p=(2 h+1)^{2}$ defines the size of the local SST neighborhood around $i$ and $h$ is set according to the Rossby radius of the study region, which states the mean size of the mesoscale ocean structures like eddies. In our study region, the Agulhas current, it approximately corresponds to $200 \mathrm{~km}$, i.e. $h=4$ for the spatial resolution of the data in this study.

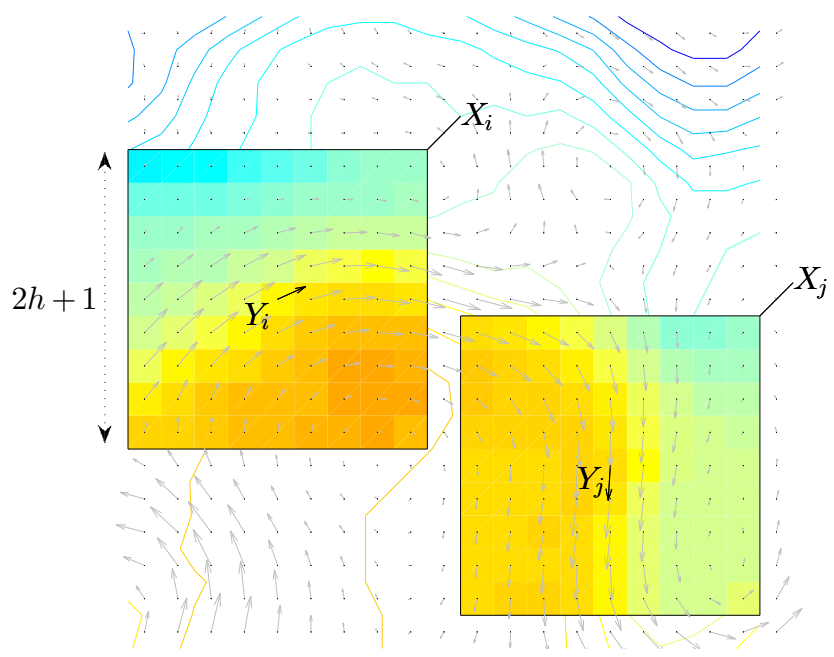

Fig. 2. Sketch of sea surface temperature patches (contour plot), noted as $\mathbf{X}$, and the corresponding zonal and meridional currents (quiver) noted $\mathbf{Y}$ at the central position.

\subsection{Latent class regression model}

Our objective is to identify $K$ different hidden surface dynamical modes from a joint set of SST patches ( $p$-dimensional vector $\mathbf{X}$ ) and zonal and meridional surface currents (2dimensional vector $\mathbf{Y}$ ). In this paper, we assume that the conditional likelihood of $\mathbf{Y}$ given $\mathbf{X}$ resorts to a mixture of Normal distributions such as

$$
p(\mathbf{Y} \mid \mathbf{X}, \boldsymbol{\theta})=\sum_{k=1}^{K} \lambda_{k} \mathcal{N}_{k}\left(\mathbf{Y} ; \mathbf{X} \boldsymbol{\beta}_{k}, \boldsymbol{\Sigma}_{k}\right)
$$

where $\mathcal{N}_{k}$ represents a multivariate Gaussian probability density function with mean $\mathbf{X} \boldsymbol{\beta}_{k}$ and covariance $\boldsymbol{\Sigma}_{k}$, and $\lambda_{k}$ is the prior probability of mode $k$. To simplify the notations, we store the overall parameters of model (4) in $\boldsymbol{\theta}=\left(\lambda_{1}, \ldots, \lambda_{K}, \boldsymbol{\beta}_{1}, \ldots, \boldsymbol{\beta}_{K}, \boldsymbol{\Sigma}_{1}, \ldots, \boldsymbol{\Sigma}_{K}\right)$. In the literature, this model is referred to as a "latent class regression" or "clusterwise regression" (cf. [11]). By construction, it imposes that $0 \leq \lambda_{k} \leq 1, \sum_{k=1}^{K} \lambda_{k}=1$ and $\boldsymbol{\Sigma}_{k}$ is positive defined. To learn model parameters $\boldsymbol{\theta}$, we resort to a classical maximum likelihood criterion and use an iterative EM procedure (cf. [12]). 


\subsection{Segmentation maps and current predictions}

We exploit the inferred model with parameters $\hat{\boldsymbol{\theta}}$ to perform a spatio-temporal segmentation of the underlying dynamical modes. More precisely, for any spatial location $s$ and time $t$, using the Bayes' theorem, we evaluate the posterior likelihood that the dynamical mode is of type $k$ such as

$$
\hat{\pi}_{k}(s, t)=\frac{\hat{\lambda}_{k} \mathcal{N}_{k}\left(\mathbf{Y}(s, t) ; \mathbf{X}(s, t) \hat{\boldsymbol{\beta}}_{k}, \hat{\boldsymbol{\Sigma}}_{k}\right)}{\sum_{l=1}^{K} \hat{\lambda}_{l} \mathcal{N}_{l}\left(\mathbf{Y}(s, t) ; \mathbf{X}(s, t) \hat{\boldsymbol{\beta}}_{l}, \hat{\boldsymbol{\Sigma}}_{l}\right)}, \forall k .
$$

Then, the pixel at location $s$ and time $t$ is assigned to the most likely dynamical mode. The estimation of the surface current at the spatial location $s$ and time $t$ is given by the fuzzy regression

$$
\hat{\mathbf{Y}}(s, t)=\sum_{k=1}^{K} \hat{\pi}_{k}(s, t) \mathbf{X}(s, t) \hat{\boldsymbol{\beta}}_{k} .
$$

\section{RESULTS}

\subsection{Remote sensing data}

As surface current data $(\mathrm{U}, \mathrm{V})$, we use the daily delayed time Maps of Absolute Dynamic Topography (MADT) produced by Collecte Localisation Satellites (CLS) available online at http://www.aviso.oceanobs.com/. This information combines the signal of several altimeters onto a $1 / 3$ degree Mercator projection grid. We use the 2004 data since four altimeters were available (Jason-1, Envisat or ERS-2, Topex/Poseidon and GFO). As SST data, we use optimally interpolated microwave SSTs provided by Remote Sensing System (RSS) available online at http://www.ssmi.com/. It combines the signal of three microwave radiometers (TMI, AMSR-E and WindSAT) which are robust to the presence of clouds. The spatial resolution is $1 / 4 \times 1 / 4$ degrees and the temporal resolution is the same as the MADT data, i.e. daily. We bilinearly interpolate the MADT data onto the SST grid. We focus on the Agulhas region between longitudes $5^{\circ} \mathrm{E}$ to $65^{\circ} \mathrm{E}$ and latitudes $30^{\circ} \mathrm{S}$ to $48^{\circ} \mathrm{S}$.

To fit the latent class regression model and learn the model parameters $\boldsymbol{\theta}$ according to the EM procedure, we build a set of joint SST patches $\left\{\mathbf{X}_{i}\right\}_{i \in\{1, \ldots, n\}}$ and surface current data $\left\{\mathbf{Y}_{i}\right\}_{i \in\{1, \ldots, n\}}$, which refer to randomly selected space-time positions in the considered time series of sea surface observations. Overall we typically consider a sample with $n=10^{5}$ observations to balance between the representativeness of the training set and the computational complexity of the EM estimation.

\subsection{Segmentation of ocean surface dynamics}

In this study, as a trade-off between the likelihood and the complexity of the model, we consider $K=4$ hidden sur- face dynamical modes, i.e. four different linear transfer functions as in Eq. (2). Then, given the estimated model parameters $\hat{\boldsymbol{\theta}}$, we determine from Eq. (5) the posterior likelihood $\hat{\pi}_{k}$ of each spatio-temporal location $(s, t)$ to be assigned to any dynamical model $k$. This leads to dynamical modes which are spatio-temporally well-segmented as illustrated in Fig. 3. The animations of the time series of these daily maps in the Agulhas current over 2004 are available here: http://tandeo.wordpress.com/communications/articles/.

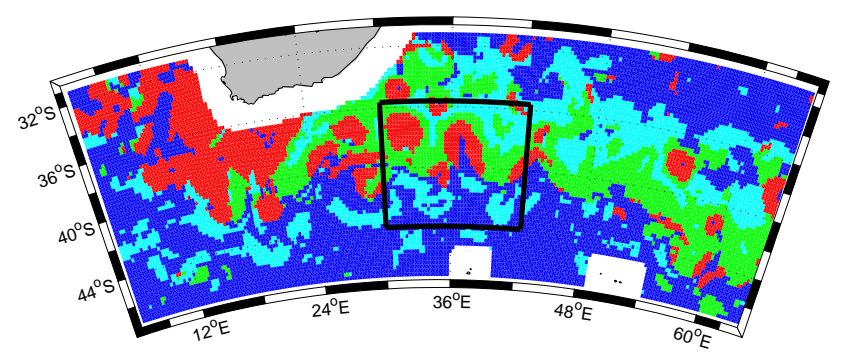

Fig. 3. Segmentation of hidden surface dynamical modes given satellite SST and surface current $(\mathrm{U}, \mathrm{V})$ data, the $1^{\text {st }}$ of January, 2004 within the Agulhas current. We use the colors red, green, cyan and blue to respectively distinguish the first, second, third and fourth dynamical modes.

These maps highlight clear spatio-temporal clusters that can be interpreted from a geophysical perspective in terms of different dynamical modes. The first dynamical mode (red) characterizes very strong current magnitude and warm waters. It is primarily associated with the main Agulhas current that flows down the East coast of Africa through the Agulhas ridge. This mode also involves mesoscale eddies, the socalled warm core Agulhas rings. An example is visible near the location $37^{\circ} \mathrm{S}, 32^{\circ} \mathrm{E}$ in Fig. 3. It corresponds to the eddy identified in the upper left corner of Fig. 1 with strong surface currents and low temperature gradients. The second cluster (green) mainly relates to the eastward Agulhas return current that hits a part of the South Atlantic current. It creates a subtropical front varying from $36^{\circ} \mathrm{S}$ to $44^{\circ} \mathrm{S}$ with strong eastward currents and middle-range SST gradients as observed in the upper part of Fig. 1. This cluster share some agreement with the SQG theory. The third (cyan) and fourth (blue) modes correspond to weaker surface currents. The third mode is characterized by mid-temperatures and westward currents whereas the fourth one involves colder temperatures and eastward currents. Let us stress that the third cluster involves large SST gradients but weak surface currents such as identified in the lower-part of Fig. 1.

\subsection{Surface current predictions}

We further investigate the geophysical consistency of the identified dynamical modes from the analysis of the surface currents predicted by the fitted latent class regression model 
in Eq. (6). In Fig. 4, we report a comparison to the MADT data, considered as a true surface current references. Overall, a good agreement is obtained between the true surface currents and the predictions of the proposed model. The global correlation coefficient is 0.76 , and can locally be very large as illustrated in Fig. 4(c), corresponding to the zone depicted in Fig. 1. As found in Fig. 3 this zone involves the four dynamical modes. The mixture model enables us to retrieve both the large warm eddy (upper left) associated with weak SST gradients, the relatively large surface currents along the large warmer SST gradients (upper part), as well as the rather weak currents along the large but colder SST front (lower part of the zone).

By contrast, if we conceal the information of the hidden dynamical modes and learn other classical models with the same $n=10^{5}$ training data, the resulting surface currents are not consistent. For example, when we fit a linear model as in Fig. 4(d), i.e. corresponding to $K=1$ in our proposed model, the single linear transfer function results in underestimating the surface currents within the warm eddy (upper left) and overestimating the currents of the colder frontal zone (lower part). We also consider a classical nonlinear approach such as the weighted 10-nearest neighbor regression in Fig. 4(e). This last model is not able to follow the warm eddy (top-left) and the estimated surface currents globally display discontinuities.

\section{CONCLUSION}

In this paper, we propose an observation-driven framework to spatially and temporally discriminate hidden dynamical modes at the surface of the Ocean. We rely on a latent class regression model. This probabilistic setting resorts to locally model the distribution of the sea surface currents $(\mathrm{U}, \mathrm{V})$ conditionally to the SST as a Gaussian mixture of hidden linear transfer functions. The statistical parameters of the model are estimated using a maximum likelihood approach. We applied the proposed methodology to the 2004 daily $1 / 4 \times 1 / 4$ degrees satellite image series of microwave SSTs and surface currents. The reported results retrieved a relevant spatiotemporal decomposition of ocean surface dynamical modes in the Agulhas region according to four dynamical modes: (i) the main Agulhas current and warm core rings characterized by strong currents and hot temperatures, (ii) the return Agulhas current with lower temperatures and currents but where the SST is dynamically coupled as in the SQG theory, (iii) local fronts regions where strong SST gradients do not seem to affect the current velocities, and (iv) a weaker dynamical mode.

Given a set of identified ocean surface dynamical modes, we show that the latent class regression model is able to make relevant predictions of sea surface currents. In particular, the four-latent class regression overcomes the results given by a linear model or classical nonlinear approaches corresponding

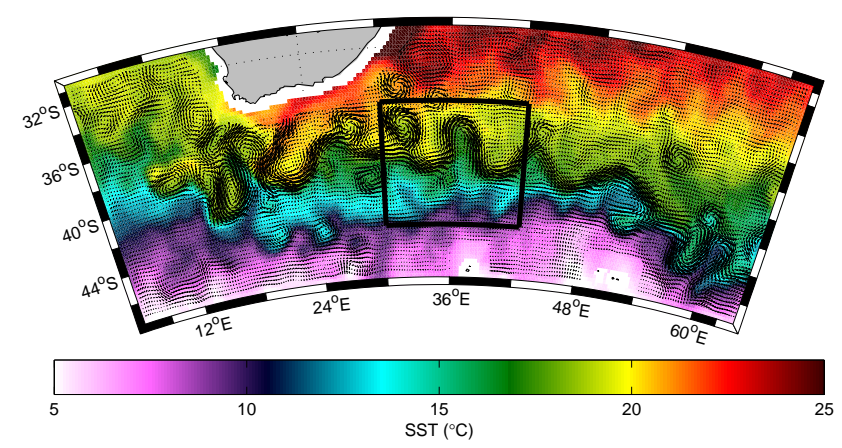

(a) True currents

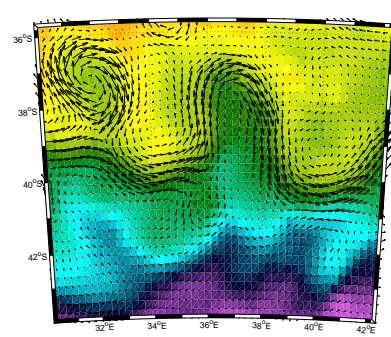

(b) True currents

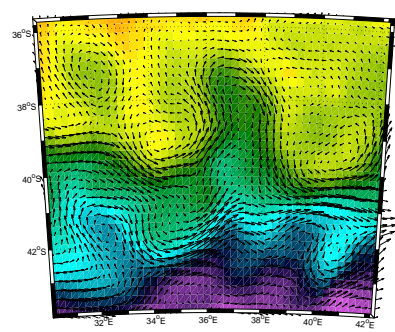

(d) Linear model

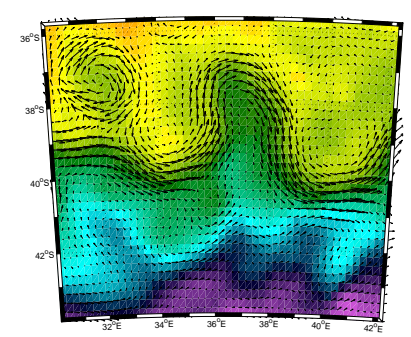

(c) Latent class regression

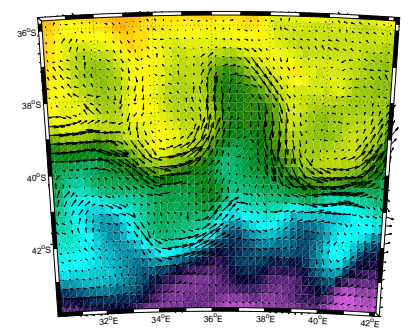

(e) Weighted k-nearest neighbor

Fig. 4. Sea surface temperature with the associated true MADT or estimated surface currents $(\mathrm{U}, \mathrm{V})$ using a linear regression, a four-latent class regression and a weighted knearest neighbor regression within the Agulhas region the $1^{\text {st }}$ of January, 2004.

to situations where we conceal the dynamical modes. This result confirms the fact that a spatio-temporal tracking of the hidden surface dynamical modes is essential to retrieve accurate surface currents given single SST fields. The latent class model presented in this paper constitutes a serious strategy to fill the gaps of satellite surface currents using abundant microwave SST data.

\section{COPYRIGHT FORMS}




\section{REFERENCES}

[1] M.M. Bowen, W.J. Emery, J.L. Wilkin, P.C. Tildesley, I.J. Barton, and R. Knewtson, "Extracting multiyear surface currents from sequential thermal imagery using the maximum cross-correlation technique," Journal of Atmospheric and Oceanic Technology, vol. 19, no. 10, pp. 1665-1676, 2002.

[2] J. Marcello, F. Eugenio, F. Marqués, A. HernandezGuerra, and A. Gasull, "Motion estimation techniques to automatically track oceanographic thermal structures in multisensor image sequences," Geoscience and Remote Sensing, IEEE Transactions on, vol. 46, no. 9, pp. 2743-2762, 2008.

[3] W. Chen, "Surface velocity estimation from satellite imagery using displaced frame central difference equation," Geoscience and Remote Sensing, IEEE Transactions on, vol. 50, no. 7, pp. 2791-2801, 2012.

[4] JH LaCasce and A. Mahadevan, "Estimating subsurface horizontal and vertical velocities from sea-surface temperature," Journal of Marine Research, vol. 64, no. 5, pp. 695-721, 2006.

[5] G. Lapeyre and P. Klein, "Dynamics of the upper oceanic layers in terms of surface quasigeostrophy theory," Journal of physical oceanography, vol. 36, no. 2, pp. 165-176, 2006.

[6] J. Isern-Fontanet, G. Lapeyre, P. Klein, B. Chapron, and M.W. Hecht, "Three-dimensional reconstruction of oceanic mesoscale currents from surface information," Journal of Geophysical Research, vol. 113, no. C9, pp. C09005, 2008.

[7] P. Klein, J. Isern-Fontanet, G. Lapeyre, G. Roullet, E. Danioux, B. Chapron, S. Le Gentil, and H. Sasaki, "Diagnosis of vertical velocities in the upper ocean from high resolution sea surface height," Geophysical Research Letters, vol. 36, no. 12, pp. L12603, 2009.

[8] U. Hausmann and A. Czaja, "The observed signature of mesoscale eddies in sea surface temperature and the associated heat transport," Deep Sea Research Part I: Oceanographic Research Papers, 2012.

[9] J. Isern-Fontanet, B. Chapron, G. Lapeyre, and P. Klein, "Potential use of microwave sea surface temperatures for the estimation of ocean currents," Geophysical research letters, vol. 33, no. 24, pp. L24608, 2006.

[10] L. Caffarelli and L. Silvestre, "An extension problem related to the fractional laplacian," Communications in partial differential equations, vol. 32, no. 8, pp. 1245 1260, 2007.
[11] W.S. DeSarbo and W.L. Cron, "A maximum likelihood methodology for clusterwise linear regression," Journal of classification, vol. 5, no. 2, pp. 249-282, 1988.

[12] A.P. Dempster, N.M. Laird, and D.B. Rubin, "Maximum likelihood from incomplete data via the em algorithm," Journal of the Royal Statistical Society. Series B (Methodological), pp. 1-38, 1977. 